\title{
Robot-assisted en bloc anterior mediastinal mass excision with pericardium and adjacent lung for locally advanced thymic carcinoma
}

\author{
Hee Chul Yang, Garrett Coyan, Matthew Vercauteren, Neha Reddy, James D. Luketich, Inderpal S. Sarkaria \\ Department of Cardiothoracic Surgery, University of Pittsburgh School of Medicine and the University of Pittsburgh Medical Center, Pittsburgh, \\ PA, USA \\ Correspondence to: Inderpal S. Sarkaria, MD, FACS. Department of Cardiothoracic Surgery, Division of Thoracic Surgery, University of Pittsburgh \\ Medical Center, Shadyside Medical Building, 5115 Centre Ave, Pittsburgh, PA 15232, USA. Email: sarkariais@upmc.edu.
}

\begin{abstract}
Robot-assisted surgery for anterior mediastinal mass resection has been increasingly adopted as an alternative method to open sternotomy and conventional video-assisted thoracic surgery. However, more evidence is needed to expand the indication of this technique to more complicated cases. We present a case of robot-assisted en bloc resection of a $7-\mathrm{cm}$ anterior mediastinal mass with pericardium and adjacent lung for thymic squamous cell carcinoma, accompanied by reconstruction of pericardium with polytetrafluoroethylene patch. In conclusion, complex anterior mediastinal mass excision is feasible with robotic thoracic surgery.
\end{abstract}

Keywords: Thymectomy; thymoma; pericardial reconstruction; robot thoracic surgery

Received: 07 May 2018; Accepted: 14 May 2018; Published: 31 May 2018.

doi: 10.21037 /jovs.2018.05.20

View this article at: http://dx.doi.org/10.21037/jovs.2018.05.20

\section{Introduction}

Although lung and pericardial invasion caused by thymic malignancy is rare, it requires en bloc surgical excision of all structures involved to achieve negative margins, and subsequent reconstructive techniques as required (1-3). In these locally advanced cases, en bloc thymectomy by median sternotomy has been considered as the standard approach. However, the evolutionary development of robotic systems and the accumulation of robotic surgery experience have allowed some experienced robotic surgeons to approach more complex and difficult cases with these techniques (4-7).

We present a case of robot-assisted en bloc resection of a large anterior mediastinal mass with pericardium and adjacent lung for thymic squamous cell carcinoma. Reconstruction of pericardium was subsequently performed with polytetrafluoroethylene (PTFE) patch using the robotic surgical system.

\section{Case presentation}

A 61-year-old male with a history of heavy alcohol drinking and smoking was referred to our hospital with an incidental finding of large anterior mediastinal mass during his regular follow-up for chronic obstructive pulmonary disease. The patient was also being managed for chronic hepatitis $\mathrm{C}$ and Child's class A liver cirrhosis at the time of consultation. The chest computed tomography (CT) images revealed approximately $7.2 \mathrm{~cm} \times 3.4 \mathrm{~cm} \times 4.5 \mathrm{~cm}$ mass in sagittal, anteroposterior, and transverse dimension, which was contiguous and in contact with the posterior cortex of the right side of the sternum, the anterior wall of the ascending aorta, and the innominate vein. The mass contained dense central calcification, and no enlarged mediastinal lymph nodes were identified (Figure 1). Laboratory findings for serum alpha-fetoprotein (AFP), beta-human chorionic gonadotropin ( $\beta-\mathrm{HCG})$, lactate dehydrogenase (LDH), and 

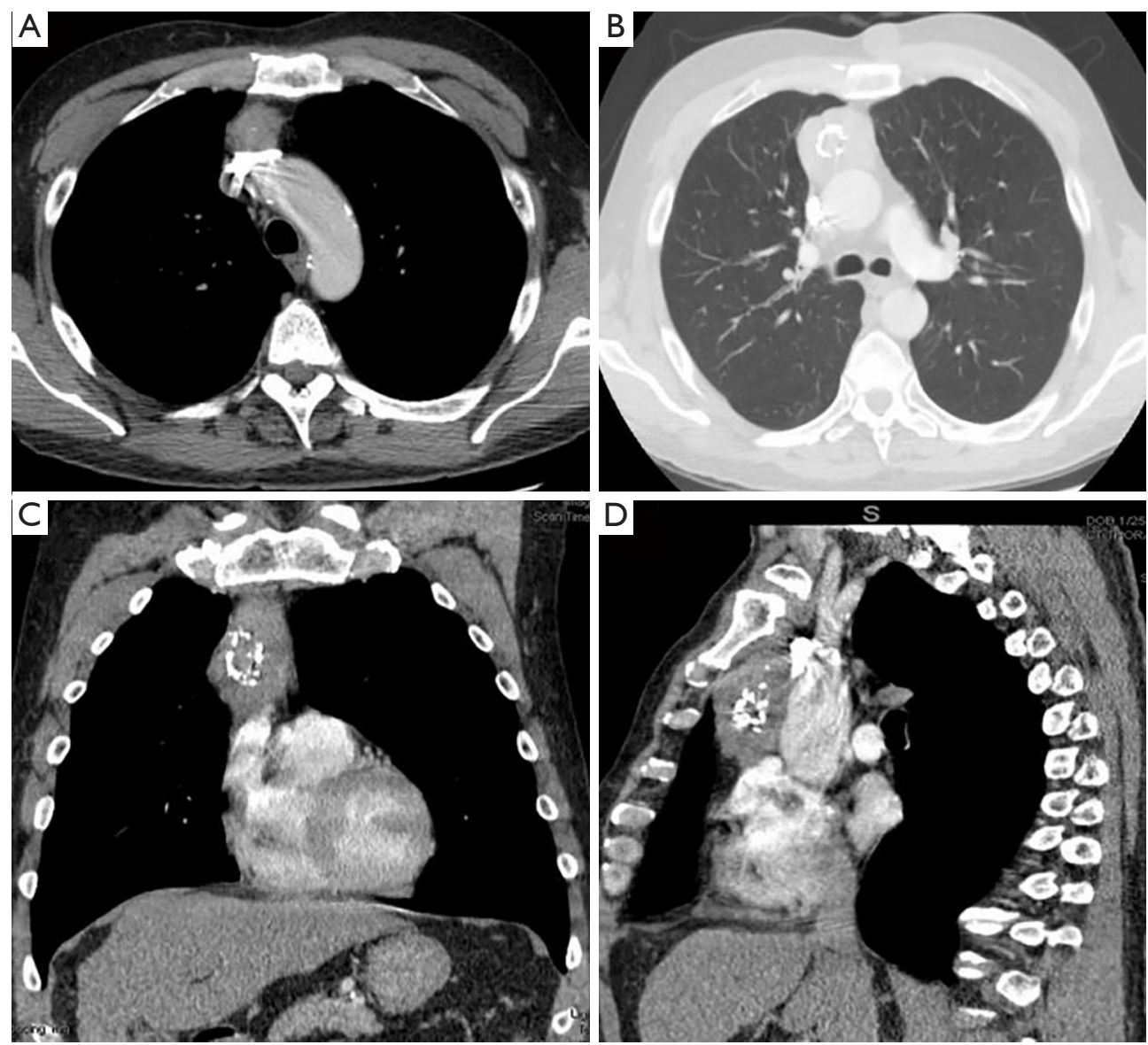

Figure 1 Chest CT-scan images. (A) Horizontal section: the superior portion of the thymic mass is lying on the left innominate vein; (B) horizontal section: the largest transverse diameter portion of the thymic mass is shown with the relationship of ascending aorta; (C) coronal section: the ovoid-elongated mass is located on the right side of the median line; (D) sagittal section: the boundaries of the thymic mass are shown between the sternum and heart. CT, computed tomography.

anti-cholinesterase antibody were all within normal limits. He was recommended to undergo a robot-assisted resection for the anterior mediastinal mass under the suspicion of thymic malignancy.

\section{Surgical technique}

After induction of general anesthesia with a double lumen endotracheal tube, the patient was positioned in the left semi-lateral decubitus position for a right-sided approach. Draping for extended total thymectomy always includes the opposite side of the chest in our practice. We chose to apply a three-arm technique using the robotic surgical system $(\mathrm{Da}$ Vinci Xi, Intuitive Surgical, Inc., Sunnyvale, CA, USA). Once the right pleural space was accessed with a $2-\mathrm{mm}$ veress needle trocar $\left(\right.$ VersaStep ${ }^{\mathrm{TM}}$, United States Surgical,
Norwalk, CT, USA), carbon dioxide $\left(\mathrm{CO}_{2}\right)$ with $8 \mathrm{mmHg}$ of pressure was infused to develop this pleural space further. The first port for the 3 -dimensional $30^{\circ}, 8 \mathrm{~mm}$ camera was made at the 7 th intercostal space (ICS) in the anterior axillary line. The other two ports were made at the $3 \mathrm{rd}$ ICS in the anterior axillary line for the left robotic arm and the 7th ICS in the mid-clavicular line for the right robotic arm respectively. A 12-mm bedside assistant port was made in the anterior axillary line of the 8th ICS just above the costophrenic recess (Figure 2).

After all the ports were placed, the robot system was advanced and docked to the ports (Figure 3). Fenestrated bipolar forceps in the left arm and Harmonic ACE $^{\circledR}$ curved shears (Ethicon Endo-Surgery, Cincinnati, OH, USA) in the right arm were mainly used during the procedure. Dissecting monopolar scissors were used when more 

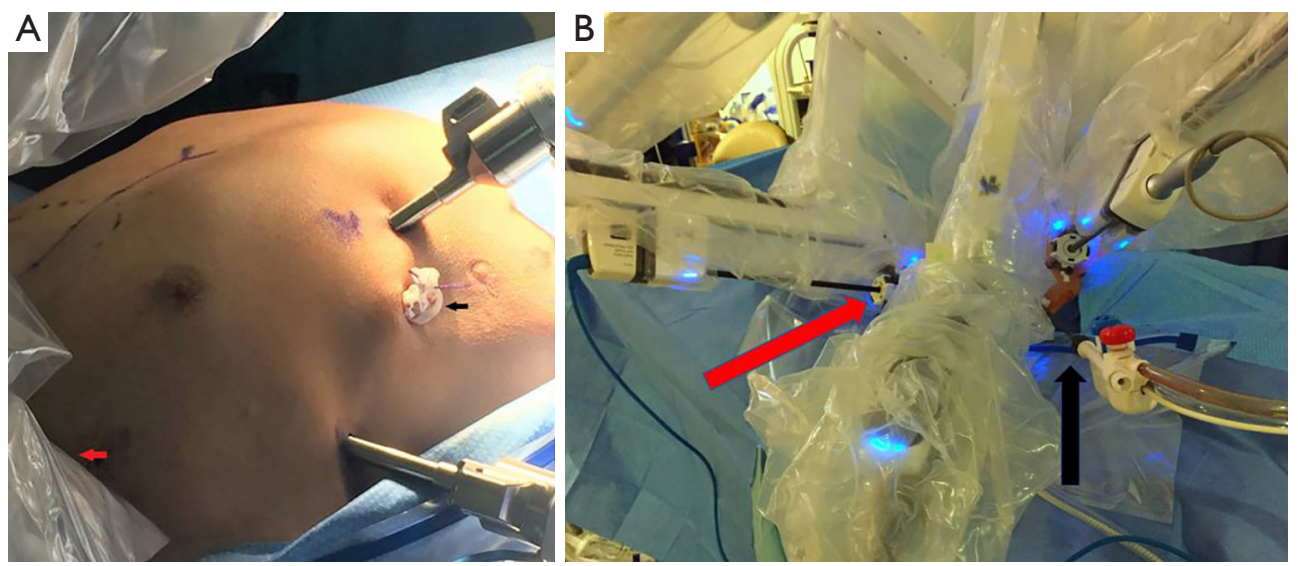

Figure 2 Port placement for the right-sided lateral approach. (A) With robot undocked; (B) with three robotic arms docked and one assistant port. Red and black arrows indicate left robotic arm and bedside assist port, respectively.

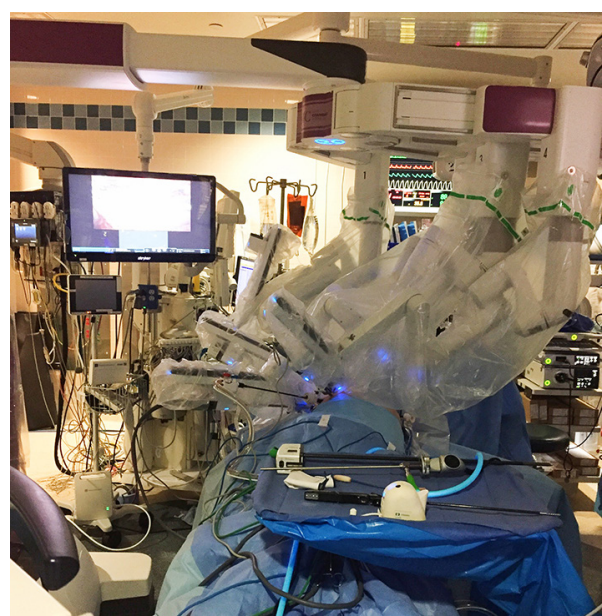

Figure 3 The da Vinci $\mathrm{Xi}^{\circledR}$ has a boom-mounted system, which enables the surgical cart to be placed at any position around the patient.

detailed dissection was required along the innominate vein. The mass was identified in the right upper anterior mediastinum and was adhered to the right upper lobe (RUL) of the lung without evidence of pleural dissemination. Wedge resection of RUL was first performed with an endoscopic stapler to take the lung en bloc with the main mass. Mobilization of the thymus was started at the anterior portion of the right phrenic nerve, which was adherent but not involved with the mass. It was continued parallel to the phrenic nerve and the superior vena cava (SVC). Next, the thymus was dissected off the pericardium from the right lower pole of thymus while moving superiorly

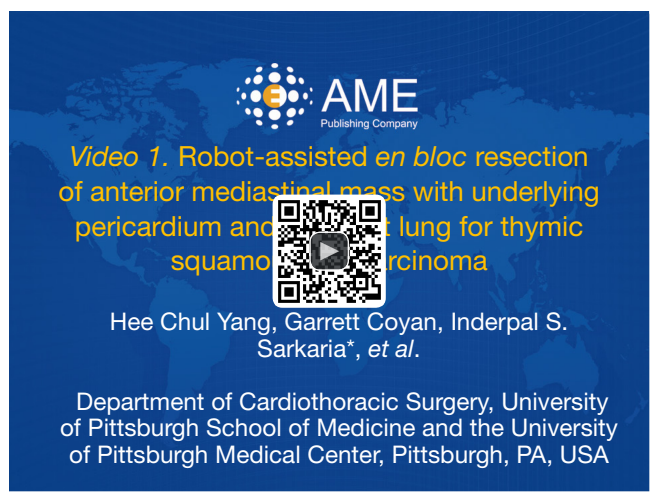

Figure 4 Robot-assisted en bloc resection of anterior mediastinal mass with underlying pericardium and adjacent lung for thymic squamous cell carcinoma (8).

Available online: http://www.asvide.com/article/view/25050

towards the mass. Clear evidence of pericardial involvement was identified near the inferior portion of the mass. The pericardium was incised and taken en bloc with the mass. The thymus was also completely mobilized medially by dividing the mediastinal pleura anteriorly along the length of the internal mammary artery and vein at the level of the sternum. The video is shown in Figure 4.

The dissection was carried out until we entered the left pleural space. A 5 -mm trocar was placed in the 5th ICS in the mid-clavicular line in the left chest, and an additional thoracoscope introduced to directly visualize the left phrenic nerve. This image was directly and simultaneously visualized by the surgeon as an additional window within the robotic console using dedicated software (TilePro, 

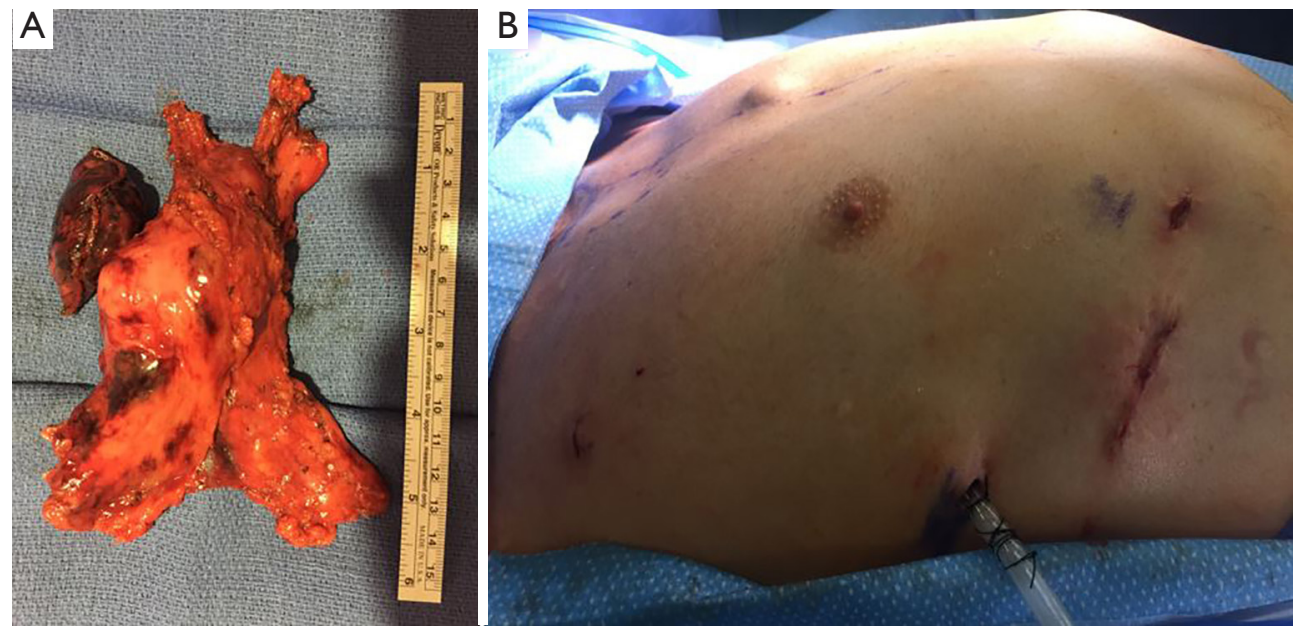

Figure 5 Specimen and postoperative wound. (A) Total thymectomy with adjacent lung and underlying pericardium; (B) the mass was extracted from the enlarged intercostal space after segmental rib resection in the assistant port site.

Intuitive Surgical, Inc., Sunnyvale, CA, USA). Thus, dissection of the left thymus was performed parallel to the left phrenic nerve under direct vision. The robotic scissors were used for fine dissection to develop the plane between the tumor and the innominate vein, which was tightly adherent to the tumor, but not directly invaded. This dissection was aided by the tenfold magnified view. Three venous branches from the innominate vein were controlled by the robotic small clip applier. Exposure and dissection of the superior thymic horns was achieved with gentle caudad traction on the mass. The mass was completely freed and placed aside in the chest. Next, circumferential biopsies were sent off for frozen section analysis and were negative for cancer.

The pericardial defect measured approximately $10 \mathrm{~cm}$ by $10 \mathrm{~cm}$. The pericardium was reconstructed with $0.1 \mathrm{~mm}$ thickness PTFE membrane (Gore ${ }^{\circledR}$ Medical Membrane, Gore \& Associates, Inc., USA) using 3-0 polypropylene suture (Prolene, Ethicon, Somerville, NJ, USA) in a running fashion using the robotic large SutureCut ${ }^{\mathrm{TM}}$ needle driver. Small pie hole cuts were made in the pericardial patch so that fluid could drain. The robot was then undocked. After a small segmental "shingle" resection of the $8^{\text {th }}$ rib through the elongated assistant port, the en bloc specimen was extracted in a retrieval bag (Anchor bag, Anchor Products Company, Addison, IL, USA) (Figure 5). A 9 Fr. pigtail catheter was placed through the port on the left chest, and a $28 \mathrm{Fr}$. chest tube was placed through the robotic camera port site in the right chest. The operative time was 6 hours 47 minutes and estimated blood loss was $20 \mathrm{~mL}$. There were no intraoperative events.

\section{Discussion}

We describe a surgical technique detailing robotic-assisted anterior mediastinal mass resection with en bloc removal of lung and pericardium, and with patch repair of the pericardial defect. Although few reports have referenced robot-assisted thymectomy with partial pericardial resection in locally advanced cases (6,9-11), they rarely describe the surgical technique and approach for these complex cases. Suda (12) provided a detailed description of the technical approach in a review article for robotic subxiphoid thymectomy with pericardial reconstruction. He postulated that thymectomy via subxiphoid approach is advantageous in visualization of bilateral phrenic nerves and upper poles. However, the subxiphoid approach is unfamiliar to many thoracic surgeons, and also has the handicap of a relatively narrow working space between the sternum and the heart. Additionally, the subxiphoid approach has limited visibility to the bilateral lower poles of thymic tissue near the hemidiaphragms. In the approach we report here, using robotic platform dedicated software, we have supplemented a 5 -mm thoracoscope into the opposite chest to directly and simultaneously visualize the contralateral phrenic nerve to overcome the drawback of lateral approach. This allows complete visualization of the lateral, superior and inferior borders of the entire anterior mediastinum, potentially allowing for more complete and aggressive resections where indicated. We found this alteration key to performing the 
pericardial reconstruction as well, as all sutures were able to be visualized from multiple angles to ensure adequate reconstruction of the pericardial defect.

Complete resection of the tumor is considered mandatory for all localized thymic malignancies. Diagnostic biopsy followed by neoadjuvant therapy before local treatment may be an alternative for tumors initially thought to be unresectable disease $(2,3)$. Several reports suggest that innominate vein invasion is a poor prognostic factor (13-15). If the invasion is strongly suspected in preoperative images, induction therapy should be considered to increase the probability of complete resection. In this case, meticulous dissection was used to develop the plane between the innominate vein and the large tumor. Division of thymic branches from innominate vein required very careful attention to avoid bleeding. The robotic system was highly advantageous in completing this step via a minimally invasive approach due to the advanced optical system and fine-scaled movements of the surgical system. This is a distinct advantage over current video-assisted thoracic surgery technologies, and we believe it is advantageous to have this advanced capability to undertake locally advanced cases. If we found that the innominate vein was involved or unable to be dissected safely, it potentially could have been treated with simple resection alone with or without reconstruction. It has been reported that the sequelae after innominate vein resection without reconstruction may be acceptable without significant long-term morbidity (16).

Robot-assisted thoracic surgery has been successfully utilized for thymectomy through either left or right-sided approaches $(17,18)$. In this particular case, we approached via the right lateral side because the lesion was mainly located on the right. However, we typically prefer the leftsided approach for extended total thymectomy in patients with myasthenia gravis or central tumors. Anatomically, the cranial thoracic portion of the right phrenic nerve is more posteriorly located in the thorax compared to the left phrenic nerve and naturally protected by SVC. Therefore, it would be more advantageous to directly visualize the left phrenic nerve, which is usually more exposed to the operative field, and with a less distinct course in the superior mediastinum.

From a long historical perspective, the extent of the application of minimally invasive surgery has continued to expand with the synergy of accumulated experiences and advanced technologies, evolving into standard of care in many cases. The development of the robotic surgical system has opened a new era of minimally invasive thoracic surgery.
We believe that robotic thoracic surgery will continue to be useful in cases such as we describe where advanced optics and detailed, wristed instrument control are essential to complete complex cases via a minimally invasive technique.

\section{Acknowledgements}

None.

\section{Footnote}

Conflicts of Interest: IS Sarkaria is a Speaker for Intuitive Surgical. The other authors have no conflicts of interest to declare.

Informed Consent: Written informed consent was obtained from the patient for publication of this manuscript and any accompanying images.

\section{References}

1. Zhao Y, Zhao H, Hu D, et al. Surgical treatment and prognosis of thymic squamous cell carcinoma: a retrospective analysis of 105 cases. Ann Thorac Surg 2013;96:1019-24.

2. Kurup A, Loehrer PJ. Thymoma and Thymic Carcinoma: Therapeutic Approaches. Clin Lung Cancer 2004;6:28-32.

3. Toker A, Sonett J, Zielinski M, et al. Standard terms, definitions, and policies for minimally invasive resection of thymoma. J Thorac Oncol 2011;6:S1739-42.

4. Solinas M, Novellis P, Bottoni E, et al. Robotic approach in case of thymoma involving the left anonymous vein: a case report. AME Case Rep 2017;1:3.

5. Okusanya OT, Hess NR, Luketich JD, et al. Technique of robotic assisted minimally invasive esophagectomy (RAMIE). J Vis Surg 2017;3:116.

6. Kuo SW, Huang PM, Lin MW, et al. Robot-assisted thoracic surgery for complex procedures. J Thorac Dis 2017;9:3105-13.

7. Qiu T, Zhao Y, Xuan Y, et al. Robotic-assisted doublesleeve lobectomy. J Thorac Dis 2017;9:E21-E25.

8. Yang HC, Coyan G, Vercauteren M, et al. Robotassisted en bloc resection of anterior mediastinal mass with underlying pericardium and adjacent lung for thymic squamous cell carcinoma. Asvide 2018;5:530. Available online: http://www.asvide.com/article/view/25050

9. Schneiter D, Tomaszek S, Kestenholz P, et al. Minimally invasive resection of thymomas with the da $\operatorname{Vinci}(\mathrm{R})$ 
Surgical System. Eur J Cardiothorac Surg 2013;43:288-92.

10. Kang CH, Hwang Y, Lee HJ, et al. Robotic Thymectomy in Anterior Mediastinal Mass: Propensity Score Matching Study With Transsternal Thymectomy. Ann Thorac Surg 2016; 102:895-901.

11. Seong YW, Kang CH, Choi JW, et al. Early clinical outcomes of robot-assisted surgery for anterior mediastinal mass: its superiority over a conventional sternotomy approach evaluated by propensity score matching. Eur J Cardiothorac Surg 2014;45:e68-e73.

12. Suda T. Robotic subxiphoid thymectomy. J Vis Surg 2016;2:118.

13. Blumberg D, Burt ME, Bains MS, et al. Thymic Carcinoma: Current Staging Does Not Predict Prognosis. J Thorac Cardiovasc Surg 1998;115:303-8;

doi: 10.21037/jovs.2018.05.20

Cite this article as: Yang HC, Coyan G, Vercauteren M, Reddy N, Luketich JD, Sarkaria IS. Robot-assisted en bloc anterior mediastinal mass excision with pericardium and adjacent lung for locally advanced thymic carcinoma. J Vis Surg 2018;4:115. discussion 308-9.

14. Utsumi T, Shiono H, Matsumura A, et al. Stage III thymoma: relationship of local invasion to recurrence. J Thorac Cardiovasc Surg 2008;136:1481-5.

15. Tseng YL, Wang ST, Wu MH, et al. Thymic carcinoma: involvement of great vessels indicates poor prognosis. Ann Thorac Surg 2003;76:1041-5.

16. Wright CD. Extended Resections for Thymic Malignancies. . J Thorac Oncol 2010;5:S344-7.

17. Deen S, Farivar AS, Louie BE. Thoracic techniques: robotic thymectomy for thymoma. Indian J Surg Oncol 2013;4:132-7.

18. Rueckert J, Swierzy M, Badakhshi H, et al. Roboticassisted thymectomy: surgical procedure and results. Thorac Cardiovasc Surg 2015;63:194-200. 